\title{
Energy Efficient Location based Routing Using DREAM Algorithm for MANET
}

\author{
Mr. Sourabh Pandey, Mr. Rajender Singh Yadav \\ MTech. Dept. of Electronics and Communication Gyan Ganga College Of Technology,Jabalpur(India) \\ Dept. of Electronics and Communication Gyan Ganga College Of Technology,Jabalpur(India)
}

\begin{abstract}
In Mobile Ad hoc Network (MANET) all the nodes are mobile in nature having limited battery capacity that is called energy. Due to the dynamic behavior of network link are not maintained for long time. All nodes in network are energy dependent and efficient energy utilization is one of the important issue in MANET. The location based DREAM (Distance routing Effect Algorithm for Mobility) protocol are maintained the location of each node on the basis of respective sender and receiver. This paper proposed a new location based energy efficient scheme with AODV (DREAM-EAODV) protocol. In this scheme energy dependent nodes are do routing with AODV protocol on the basis of location based protocol DREAM. Nodes in network are not intimated about their energy status, for that remove the suddenly link breakage. If the nodes in network are know about the energy status and also about the status of location of receiver that reduces the energy consumption. If the node ia apart of communication then in that case no link is break but if not then break. The main aim of proposed scheme is to enhance the energy utilization in network. Simulation has done by using ns-2 simulator and reduces energy consumption. The performance is measure on the basis of performance parameter like Normal Routing Load, Packet Delivery Ratio.
\end{abstract}

Index Terms: - MANET, energy, AODV, DREAM, routing

\section{INTRODUCTION}

The Ad Hoc Networks are wireless networks characterized by the absence of fixed infrastructures. The main aim of mobile ad- hoc network (MANET) [1] is to support robust and efficient operation in mobile wireless networks. MANET consists of mobile nodes which form a spontaneous network without a need of fixed infrastructure. It is an autonomous system in which mobile hosts connected by wireless links are free to move randomly and often act as routers at the same time. Hence, it forms multi-hop network. The ad-hoc networks are finding more importance likely due to the features that they can be easily deployed as well as reconfigured. This allows the use of this kind of network in special circumstances, such as disastrous events, the reduction or elimination of the wiring costs and the exchange of information among users independently from the environment. The applications for MANETs are ranging from large-scale, mobile, highly dynamic networks, to small, static networks that are constrained by power sources [2]. It can be used in military communication, commercial sectors like disaster management, emergency operations, wireless sensor networks, etc.

In the routing protocol design of mobile nodes, many issues need to be considered in order to offer many important properties such as scalability, QoS support, security, low power consumption and so on. In this chapter we focus on the energy issues facing some important aspects going from the energy model definition for the computation of the energy consumption to energy-aware metrics definition and routing protocol design. If a network composed of mobile nodes communicating using a wireless radio and where each node can communicate with each other using the other mobile nodes as relay nodes is applied in a communication system, many challenging design issues need to be addressed. MANET technology became, in the last years, more commercial in comparison with the past where it was used for military purpose and this implies more additional features to offer to the end- user with particular reference to quality of service, security and to node lifetime duration.

\section{DREAM IN MANET}

DREAM protocol in MANET are requires the information about geographical location of the nodes in network. This location information can be determined by using Global Positioning System (GPS) [3]. By using location information, DREAM protocol limits the search for a new route to a smaller request zone of the ad hoc network.

\section{RELATED WORK}

The previous works that was done in this field are mentioned in this section several energy-efficient techniques are proposed to reduce energy consumption in MANET. These techniques use energy aware metrics to establish a path in a network. These metrics are residual energy, transmission power or link distance. 
Nivedita and Radhika [4] proposed a scheme for controls the transmission power of a node according to the distance between the nodes. It also includes energy information on route request packet and selects the energy efficient path to route data packets. LAR1 protocol uses location information of a node for setting the path from source to destination. LAR1 protocol consists of source location and destination location information. ELAR1VAR protocol improves the network lifetime by reducing energy consumption by $20 \%$ for dense and mobile network while maintaining the packet delivery ratio above $90 \%$.

Morteza Maleki, Karthik Dantu, and Massoud Pedram in [5] have proposed a new power-aware sourceinitiated (on demand) routing protocol for mobile Ad-hoc networks that increase the network lifetime up to $30 \%$. A greedy policy was applied to fetched paths from the cache to make sure no path would be overused and also make sure that each selected path has minimum battery cost among all possible path between two nodes. Poweraware Source Routing (PSR) has taken care of both the node mobility and the node energy depletion that may cause a path to become invalid.

Energy-based Route Discovery Mechanism in Mobile Ad Hoc Networks [6] selects the route which has lowest energy cost in the network. The energy cost represents energy consumption of the network in order to prolong all connections between source and destination nodes. The energy cost is calculated using realistic energy consumption modelling which is used the channel quality to decide whether each packet is successfully received. Location aided Energy-Efficient Routing protocol (LEER) protocol finds out the all possible paths from source to destination and selects minimum energy path to route the packets [7]. The selection of next hop node is based on whether it is situated near to destination than to source as well as transmit power of that node.

An energy aware routing scheme in location based ad-hoc network has proposed by Jangsu Lee, Seunghwan Yoo and Sungchun KimIn [8]. This method modifies the LAR protocol in which the virtual grid is applied to ad hoc network region and high energy node is selected as header for each grid which communicates information about nodes in that particular grid. The transmit power of nodes is adjusted according to the distance between them. The next hop node will be selected based on transmit power and its distance from the destination.

Nen-Chung Wang and Si-Ming Wang [9] have proposed a scheme which decides the baseline line between the source node and the destination node, for route discovery. The next hop is then selected based on baseline by broadcasting the request packets in request zone. The neighboring node with the shortest distance to the baseline is chosen as the next hop node. This method reduces control overheads by finding a better routing path than LAR scheme. They have proposed a partial reconstruction process for maintaining broken links of routing path.

Arthi Kothandaraman has proposed a protocol which based on transmission power control [10]. It varies the transmission range of a node to exclusively accommodate an independent node's neighbor set. This purely distributed as well as protocol independent scheme and preserves connectivity, and allows low power transmissions.

Energy Efficient Location Aided Routing (EELAR) Protocol [12] was developed on the basis of the Location Aided Routing (LAR) [11]. EELAR makes significant reduction in the energy consumption of the mobile node batteries by limiting the area of discovering a new route to a smaller zone. Thus, control packet overhead is significantly reduced. In EELAR, a reference wireless base station is used and the network's circular area centered at the base station is divided into six equal sub-areas. During route discovery, instead of flooding control packets to the whole network area, they are flooded to only the sub-area of the destination mobile node. The base station stores locations of the mobile nodes in a position table. EELAR protocol makes an improvement in control packet overhead and delivery ratio compared to AODV, LAR, and DSR protocols.

$\mathrm{Li}$ et al proposed the Online Max-Min (OMM) power-aware routing protocol [13] for wireless ad-hoc networks dispersed over large geographical areas to support applications where the message sequence is not known. This protocol optimizes the lifetime of the network as well as the lifetime of individual nodes by maximizing the minimal residual power, which helps to prevent the occurrence of overloaded nodes. In most applications that involve MANETs, power management is a real issue and can be done at two complementary levels (1) during communication and (2) during idle time. The OMM protocol maximizes the lifetime of the network without knowing the data generation rate in advance. The metrics developed showed that OMM had a good empirical competitive ratio to the optimal online algorithm that knows the message sequence and the maxmin achieves over $80 \%$ of the optimal node lifetime (where the sender knows all the messages ahead of time) for most instances and over $90 \%$ of the optimal node lifetime for many problem instances.

The Power-aware Localized Routing (PLR) protocol [14] is a localized, fully distributed energy-aware routing algorithm but it assumes that a source node has the location information of its neighbours and the destination. PLR is equivalent to knowing the link costs from the source node to its neighbours, all the way to the destination. Based on this information, the source cannot find the optimal path but selects the next hop through which the overall transmission power to the destination is minimized. 


\section{PPROBLEMSTATEMENT}

Mobile Ad Hoc networks are power constrained since nodes operate with limited battery energy. Due to lack of energy and location awareness most of the energy of mobile nodes are wasted and following problems occurs

1. Suddenly loss of session.

2. Due to maximize the loss of packets.

3. Decreases network survivability and connectivity.

4. Route Overhead is Maximum.

\section{PROPOSED WORK}

Here we design the algorithm for energy aware with AODV a routing after that we follow next algorithm for destination location estimation, here very first we create mobile node and then set all parameter like routing protocol as AODV and each layer header and initial energy of node and then broadcast the routing packet, that time we check next hop information like current energy and connectivity of next neighbour node if current energy is sufficient and next node is found so we add this node into the routing table and send routing packet till the destination reach condition through above mechanism if destination found then destination node reply through routing acknowledgement packet with their location information to the source node, and after that sender node send's actual data packet to the destination. But certain time communicator and intermediate node move due to mobility nature and existing route break down so that case we apply DREAM module and minimize routing overhead of the network that also minimize energy consummation as compare to other existing routing protocol. The proposed algorithm for routing with DREAM protocol are as follows:-

Set node $=\mathrm{M} ; / / \mathrm{m}$ number of mobile node

Set Sender $=\mathrm{S} ; \quad / / \mathrm{s} € \mathrm{~m} \mathrm{~s}$ sender that belong into $\mathrm{m}$ node

Set receiver $=\mathrm{R} ; / / \mathrm{r} € \mathrm{~m} \mathrm{~s}$ receiver that belong into $\mathrm{m}$ node

Set protocol $=$ AODV; $\quad / /$ Routing protocol

Location Protocol $=$ DREAM // For location information of nodes.

Set $\mathrm{RR}=550 \mathrm{~m} \quad / /$ maximum radio range of node

Set initial energy $=I_{i e} \quad / /$ set each node different initial energy

Route_RRE(S,R,M) // broadcast route request packet

If $(\mathrm{RR}<=550$ \& \& nexthop=="true")

$\{$ next hop find ;

route-table $=$ create route table;

Work until destination search;

If (more than one route $\mathrm{S}$ to $\mathrm{R}$ )

\{find (shortest path)

\{Shortest path find Send (R,S, route table, ACK) // sends acknowledgement

\section{SIMULATION ENVIRONMENT}

To evaluate the performance of proposed E-AOMDV protocol, we present simulations using network simulator 2 (NS2-2.31) [15]. The operation system is windows 7. NS-2 are not support windows then install 'cygwin' to provide linux environment in windows. The computer configuration is listed as following: CPU: i 1.86G, Memory: 2G, Hard disc: 250G. The simulation parameters are shown in table 1.

TABLE I. SIMULATION PARAMETERS

\begin{tabular}{|l|l|}
\hline Simulator Used & NS- 2.31 \\
\hline Number of nodes & 50 \\
\hline Dimension of simulated area & $800 \mathrm{~m} \times 800 \mathrm{~m}$ \\
\hline Routing Protocol & AODV \\
\hline Simulation time & $100 \mathrm{sec}$. \\
\hline Location protocol & DREAM \\
\hline Traffic type (TCP \& UDP) & CBR $(3 \mathrm{pkts} / \mathrm{s})$ \\
\hline Packet size & 512 bytes \\
\hline Number of traffic connections & 10 \\
\hline Node movement at maximum Speed & random \& $20 \mathrm{~m} / \mathrm{s}$ \\
\hline Transmission range & $250 \mathrm{~m}$ \\
\hline Transmission energy & 1.5 Joules \\
\hline Receiving energy & 1.0 Joules \\
\hline Sense emergy & .0175 Joules \\
\hline
\end{tabular}




\section{Performance metrics}

We use four metrics to evaluate the performance of the routing protocols, in which the first two metrics are the most important for best effort route and transmit protocols.

Packet delivery ratio: or packet throughput, the fraction of the data packets delivered to destination nodes to those sent by source nodes.

Average end-to-end latency: the average time taken by the data packets from sources to destinations, including buffer delays during a route discovery, queuing delays at interface queues, retransmission delays at MAC layer and propagation time.

Routing packet overhead: the ratio of the number of control packets (including route request/reply/update/error packets) to the number of data packets.

Throughput: Number of packets sends or receives in per unit of time in network.

\section{SIMULATION RESULTS}

This section evaluated the results in case of previous EAODV case and proposed location based EAOMDV case. The performances of proposed scheme are better than previous

NAM Scenario of network

The NAM (Network AniMator) represents the number of nodes and the communication among them. In this NAM the colour code represents the different energy level of nodes. Here the black colour represents the sufficient energy level, yellow represents the $50 \%$ of energy with remaining out of total energy of node and red colour represents the danger level of nodes means only some amount of energy are left in nodes.

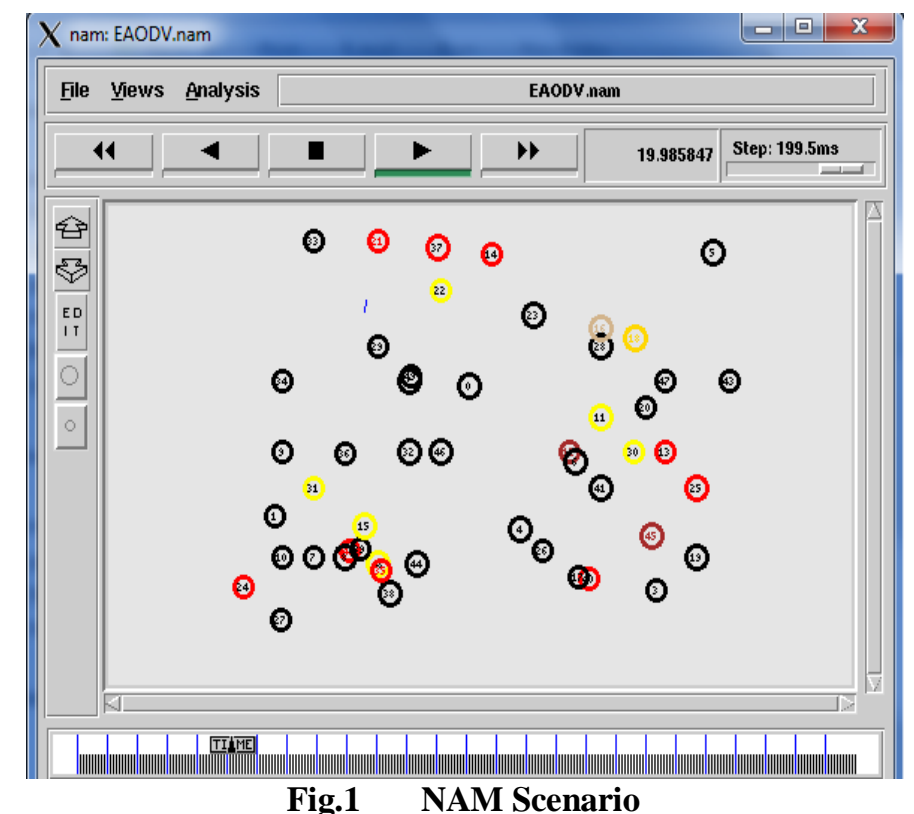

\section{Overhead Analysis in case of EAODV and proposed EAODV}

Routing load analysis is one of the important performance parameter to analyze the network performance and lot of node energy is wasted for connection establishment because if the sender sends more routing packets in that case the more energy of node are wasted and the receiver is also busy to reply them, by that the data delivery are affected and energy consumption increases. Now in this graph the routing load in previous scheme are too much because in normal energy based routing each node has do the routing after connection establishment and this procedure call again and again because sender are no aware about the location of receiver. But in proposed scheme the sender are aware about the location of receiver by that the routing packets are only deliver at time of about $60 \mathrm{sec}$. after that no routing packets are deliver in network because every sender is know about the location of receiver. In previous scheme about 1900 routing packets are used for connection establishment in between sender and receiver but in proposed scheme only 1200 packets are used. It means that the about 4000 packets are unnecessary deliver in network for that consumes lot of energy that is completely wasted but in proposed scheme this energy is saved. 


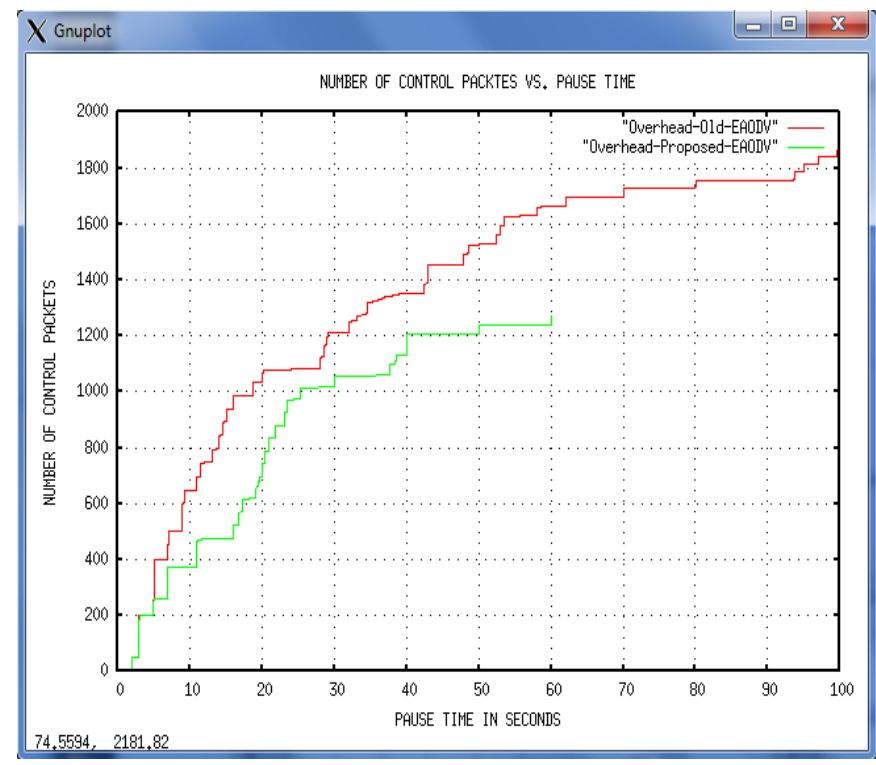

Fig.2 Routing over head Analysis

\section{Throughput Analysis in case of EAODV and proposed EAODV}

Throughput analysis are measured to evaluate the number of packets are send or receive in per unit of time. Throughput is measure to evaluate the sender packet delivery in per unit of time. This graph represents the throughput in case of old EAODV and proposed EAODV. The throughput in case of proposed AODV with location based DREAM are more as compare to previous because the location based DREAM protocol are improves the routing capability of AODV protocol by that the energy consumption in network are reduces the life of nodes are enhanced. Here we clearly notice that in case of previous scheme the maximum throughput is about 800 packets per second but in case of proposed scheme the maximum number of packets are about 1200 are sends in per unit of time in network. The reason of throughput enhancement is only that all nodes are in network are aware about the location status of neighbour.

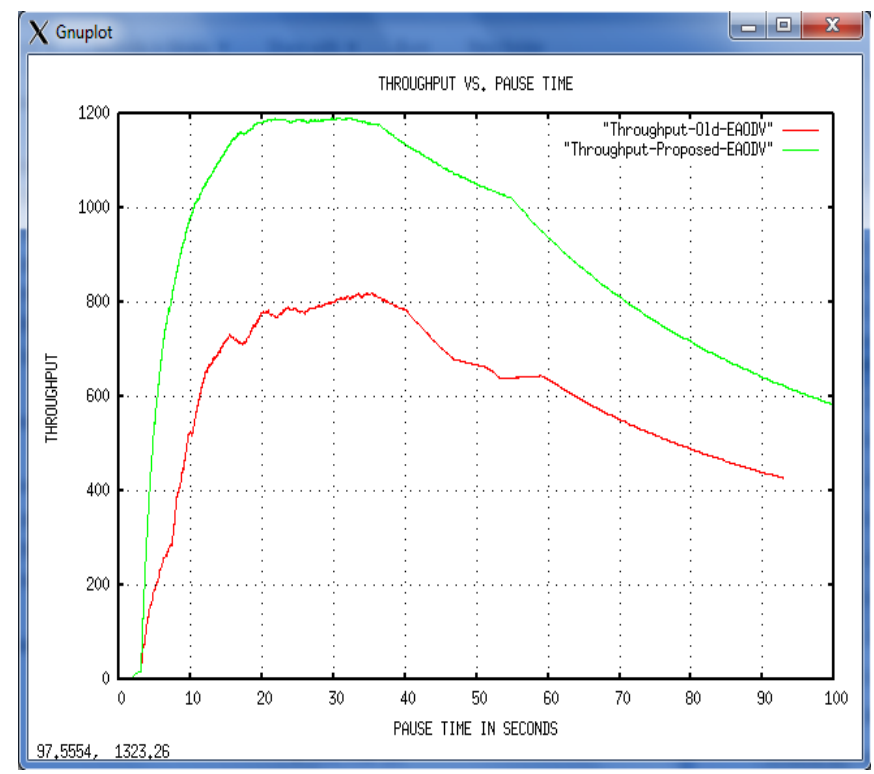

Fig. 3 Throughput Analysis

\section{Packet delivery ratio (PDR) Analysis in case of EAODV and proposed EAODV}

Packet delivery Ratio (PDF) is the percentages of data packets are successfully received in network. But this factor are completely depend on the number of packets are send and receive in network. If the less number of data packets are deliver and most of them are received it means the PDF are too good. In this graph the PDF of previous scheme are of about $96 \%$ but in case of proposed energy with DREAM scheme the PDF is about $98 \%$ because of efficient utilization of node energy. In proposed scheme at the time of about $4 \mathrm{sec}$. the value of PDF are reaches to $100 \%$ after that at time $5 \mathrm{sec}$. the PDF of previous scheme are slightly equal but at $10 \mathrm{sec}$. and after 
that the PDF are continuously increases. Now in case of previous scheme the noticeable thing is the PDF value is only measurable up to 92 seconds. It means after that no packet is received in network due to lack of energy.

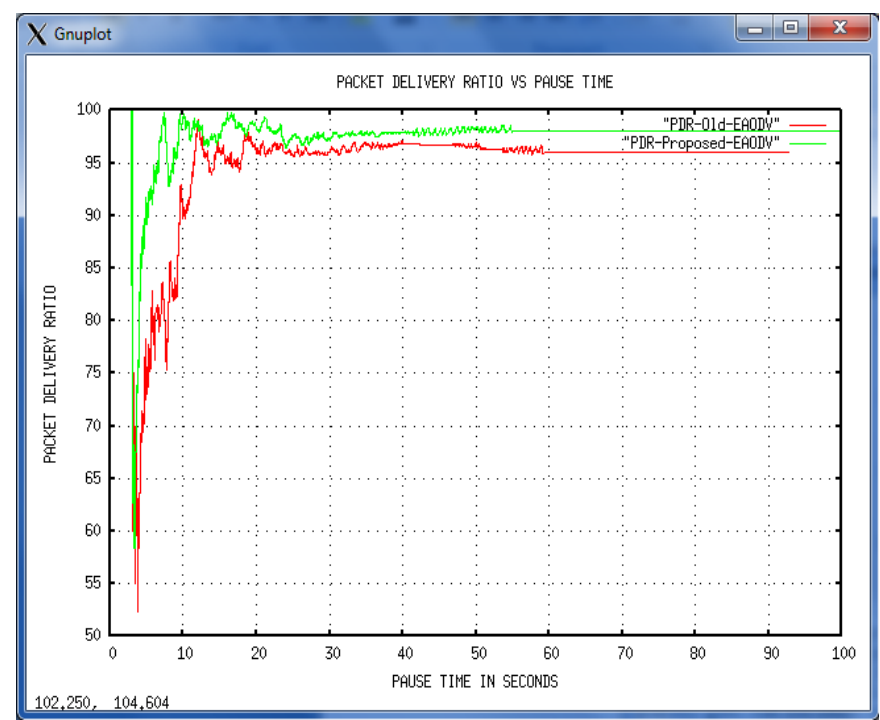

Fig. 4 PDR Analysis

\section{Rate Analysis in case of previous and proposed scheme}

The rate analysis are represents the number of bits transmission in per second in network. The table 2 represents the rate analysis in case of previous and proposed location based scheme. Here the rate analysis of proposed scheme are showing the improvement in average rate performance it means if the nodes are in network are aware about the location then definitely the rate performance improves that reduces energy consumption and enhance network life time.

TABLE II. RATE ANALYSIS

\begin{tabular}{|c|c|c|c|c|}
\hline $\begin{array}{c}\text { Source/ } \\
\text { Destination }\end{array}$ & $\begin{array}{c}\text { Start } \\
\operatorname{Time}(\mathbf{m} / \mathbf{s})\end{array}$ & $\begin{array}{c}\text { End } \\
\operatorname{Time}(\mathbf{m} / \mathbf{s})\end{array}$ & $\begin{array}{c}\text { RATE/Kbps } \\
\text { Old }\end{array}$ & $\begin{array}{c}\text { RATE/Kbps } \\
\text { Proposed }\end{array}$ \\
\hline 35 & 3 & 59.100619 & 17 & 18 \\
\hline 18 & 32.352737 & 36.963942 & 4 & 37 \\
\hline 37 & 11.092463 & 11.51567 & 47 & 34 \\
\hline 11 & 3 & 36.770377 & 0 & 37 \\
\hline 21 & 3.106976 & 59.182804 & 16 & 18 \\
\hline 12 & 3 & 99.95 & 2 & 2 \\
\hline 30 & 2 & 29.45 & 2 & 2 \\
\hline 22 & 7.415995 & 35.34485 & 35 & 30 \\
\hline 42 & 5 & 88.065631 & 0 & 2 \\
\hline 16 & 5 & 36.65569 & 32 & 22 \\
\hline \multicolumn{3}{|c|}{ AVARAGE RATE } & 15.5 & 20.2 \\
\hline
\end{tabular}

VIII. CONCLUSION \& FUTURE WORK

In this paper we proposed an efficient routing scheme with location based (DREAM) protocol that improves the utilization of node energy. In DREAM, each node records location information in a Location Table. If any node has a part of communication then in that case it first completes communication with energy alert in between source to destination. The request packet is broadcasted in a request packets are delivered on the basis of location to determine the next broadcasting node. The neighboring node with the shortest distance is chosen as the next broadcasting node. In this paper we compare the performance of Energy based AODV (E-AODV) protocol and proposed (Location based routing (DREAM-EAODV) scheme. The presence of location information are reduces the unnecessary flooding of packets in network and by that the energy of nodes that flooding packets are wasted in without location based routing. If the nodes in network are know about the energy status and also about the status of location of receiver that reduces the energy consumption. In our simulation experiments, we simulated the normal routing load, packet delivery ratio and most important energy utilization of nodes which 
take part in routing. Now results are clearly show that the performance and energy utilization are more improved then previous scheme by that the life of network are also increases.

In future analyze the energy loss in case of attack and also try to analyze the energy consumption in WiMAX technology and compare their results with WiFi technology.

\section{REFERENCES}

[1] Imrich Chlamtac, Marco Conti \& Jennifer J.-N. Liu, (2003) "Mobile ad hoc networking: imperatives and challenges", Ad-hoc Networks, Elsevier, pp.13-64.

[2] Jun-Zhao Sun, (2001) "Mobile Ad Hoc Networking: An Essential Technology for Pervasive Computing", International Conference on Info-tech and Info-net, Vol. 3, pp316-321.

[3] G. Dommety \& R. Jain, (1996) "Potential networking applications of global positioning systems (GPS)", Tech. Rep. TR-24, CS Dep., The Ohio State University.

[4] Nivedita N. Joshi and Radhika D. Joshi, "Variable Range Energy Efficient Location Aided Routing For MANET", CCSEA-2011, CS \& IT 02, pp. 321-333, 2011.

[5] Niranjan Kumar Ray \& Ashok Kumar Turuk, "Energy Efficient Techniques for Wireless Ad Hoc Network", International Joint Conference on Information and Communication Technology, pp.105-111, 2010.

[6] J. Kanjanarot, K. Sitthi, and C \& Saivichit, "Energy-based Route Discovery Mechanism in Mobile Ad Hoc Networks", ICA0T2006, pp1967-1972, 2006.

[7] Dahai Du \& Huagang Xiong, "A Location aided Energy-Efficient Routing Protocol for Ad hoc Networks", 19th Annual Wireless and Optical Communications Conference (WOCC), pp1-5, 2010.

[8] Jangsu Lee, Seunghwan Yoo \& Sungchun Kim,"Energy aware Routing in Location based Ad-hoc Networks", Proceedings of the 4th International Symposium on Communications, Control and Signal Processing (ISCCSP), pp. 3-5, 2010.

[9] Nen-Chung Wang \& Si-Ming Wang, "An Efficient Location-Aided Routing Protocol for Mobile Ad Hoc Networks", 11th International Conference on Parallel and Distributed Systems (ICPADS'05), Vol. 1, pp335-341, 2005.

[10] Arthi Kothandaraman,"An Energy-Efficient Transmission Power Control Protocol for Cooperative Robotics", Master Thesis, Auburn University, Alabama, pp.1-63, 2008.

[11] Y. Lia, X. Chengb and W. Wuc, "Optimal Topology Control for Balanced Energy Consumption in Wireless Networks", J. Parallel and Distributed Computing, vol. 65, no. 2, pp. 124 - 131, February 2005.

[12] M. Mohammed, "Energy Efficient Location Aided Routing Protocol for Wireless MANETs", International Journal of Computer Science and Information Security, vol. 4, no. 1 \& 2, 2009.

[13] Q. Li, J. Aslam, D. Rus, "Online Power Aware Routing", Proceedings of International Conference on Mobile Computing and Networking (MobiCom'2001), 2001.

[14] I. Stojmenovic and X. Lin, "Power Aware Localized Routing in Wireless Networks", IEEE Transactions on Parallel and Distributed Systems, vol. 12, no. 11, pp. 1122-1133, November 2001.

[15] The CMU Monarch Project, "The CMU Monarch Extensions to the NS Simulator",URL: http://www.monarch.cs.cmu.edu/. Page accessed on February 20th, 2010. 